\title{
Potencial da casca da laranja como biossorvente alternativo para remoção de metais pesados em águas residuais
}

Wallas Douglas de Macêdo Souza* (Mestrando do PPGCN na Universidade do Estado do Rio Grande do Norte - UERN);

Janete Jane Fernandes Alves (Prof. Ad. do DQ e do PPGCN na Universidade do Estado do RN - UERN);

Thiago Mielle Brito Ferreira Oliveira (Prof. do PPGCN na Universidade do Estado do RN - UERN);

Daniele da Silva Oliveira (Mestranda do PPGCN na Universidade do Estado do RN - UERN).

*Email: wallasdouglas_wdms@hotmail.com

Resumo:

A poluição dos recursos hídricos e os respectivos impactos socioambientais constituem motivo de preocupação mundial, em virtude da ascendente escassez deste recurso associada ao elevado custo energético e financeiro atrelado ao seu tratamento. Um dos poluentes mais preocupantes e frequentes são os metais pesados, por serem altamente tóxicos para a saúde dos seres vivos e capazes de inviabilizar a utilização da água para diferentes fins. Como alternativa, várias técnicas de tratamento têm sido propostas para a remoção de metais de águas naturais, com destaque para as tecnologias de adsorção por troca iônica. A utilização de biossorventes obtidos a partir da casca e do mesocarpo de diferentes frutos (laranja, limão, banana, fibra de coco verde, entre outros), tem mostrado resultados bastante promissores. O presente trabalho apresenta uma revisão acerca da viabilidade da casca da laranja no processo de biossorção, por conta do alto potencial da biomassa não comestível deste fruto para o processo de troca iônica. Em linhas gerais, estudos revelam que a casca da laranja pode ser utilizada em substituição ao carvão ativado comercial, que possui alto custo, mas que ainda é a técnica mais empregada para a remoção de metais em águas contaminadas. A modificação química da casca da laranja também tem mostrado excelentes resultados para a adsorção dos íons metálicos, porém estudos toxicológicos ainda necessitam ser realizados acerca dos riscos de contaminação com compostos secundários.

Palavras-chave: Metais pesados; Troca iônica; Casca da laranja; Biossorção.

Espaço reservado para organização do congresso.

\section{Metais pesados e implicações ambientais}

A falta de planejamento durante o processo de ocupação do solo acarretou sérios impactos ao meio ambiente, particularmente aos recursos hídricos. Vários poluentes vêm sendo constantemente difundidos pelas reservas, por meio de diferentes fontes antrópicas, limitando seus múltiplos usos. Entre os mais tóxicos, destacam-se os metais pesados, corantes, pesticidas, derivados fenólicos, fármacos, produtos de higiene pessoal, entre outros (KYZAS; KOSTOGLOU, 2014). Uma vez dispersos no meio ambiente, esses poluentes podem atingir toda a cadeia trófica, sendo que aqueles que se encontram no topo da cadeia alimentar, como é o caso dos seres humanos, estão mais propensos à exposição dos níveis mais elevados (ANASTOPOULOS; KYZAS, 2015). Muitos pesquisadores têm investido esforços para compreender as diferentes vias de acesso e os mecanismos de distribuição dos metais pesados em 
águas para consumo humano, pelo seu elevado potencial bioacumulador e biomagnificador (HE; CHARLET, 2013; KEDZIOREK et al., 2013; WU et al., 2014; RAZAK et al., 2015).

Os efluentes domésticos e industriais estão entre as principais fontes de metais, a exemplo do chumbo $(\mathrm{Pb})$, cobre $(\mathrm{Cu})$, cádmio $(\mathrm{Cd})$, zinco $(\mathrm{Zn})$ e níquel $(\mathrm{Ni})$, cromo $(\mathrm{Cr})$ e mercúrio $(\mathrm{Hg})$ (FU; WANG, 2011; SAKA; SAHIN; KÜÇÜK, 2012). O Pb possui diversos efeitos no organismo humano, desde neurológicos até cardiovasculares (MOREIRA; MOREIRA, 2004). O excesso de $\mathrm{Cu}$ no organismo humano leva a sua deposição no fígado e subsequentes vômitos, problemas respiratórios, entre outros (AKAR et al. 2009; BILAL et al. 2013) . Já o Cd, a exposição crônica pode levar à disfunção renal, bronquite, doenças gastrintestinais e até à morte (FU; WANG, 2011). No caso do Zn, quando em altas concentrações em um ambiente pode levar a dores de estômago, distúrbios pulmonares, febre, retardo do crescimento e até mesmo câncer (CAPRARESCU et al., 2015). Em pequenas quantidades o Ni é essencial, porém em altas concentrações aumentam-se as possibilidades de desenvolver câncer de pulmão, nariz, próstata, entre outras complicações (ALOMÁ-VICENTE et al., 2013). Apesar do Cr (III) ser menos tóxico que o $\mathrm{Cr}(\mathrm{VI})$, à exposição prolongada pode causar problemas como alergias na pele e câncer (SOUZA et al., 2012). A grande exposição ao Hg é capaz de afetar o sistema nervoso e renal, sendo a "doença de Minamata" um dos casos mais conhecidos mundialmente á respeito desses efeitos (WU et al., 2014; KUSHWAHA et al., 2015). Em um efluente, os metais podem ser encontrados tanto na forma de íons-livres quanto como complexos organo-minerais solúveis e/ou adsorvido às partículas sólidas (PIETROBELLI, 2007). No Quadro 1, encontram-se os metais frequentemente encontrados em diferentes setores industriais, cuja prioridade de recuperação considerou o risco ambiental, taxa de esgotamento de reserva e a combinação de ambos os fatores.

Quadro 1- Metais pesados encontrados nos efluentes de alguns setores industriais.

\begin{tabular}{|c|c|c|c|}
\hline Indústria & Metais & \multicolumn{2}{|c|}{ Prioridade de recuperação } \\
\hline $\begin{array}{l}\text { Mineração } \\
\text { Galvanoplastia }\end{array}$ & \multirow{3}{*}{$\begin{array}{l}\mathrm{Cu}, \mathrm{Zn}, \mathrm{Pb}, \mathrm{Mn}, \mathrm{U}, \mathrm{Cr}, \mathrm{As}, \mathrm{Se} \\
\mathrm{Cr}, \mathrm{Ni}, \mathrm{Cd}, \mathrm{Zn} \\
\mathrm{Cu}, \mathrm{Zn}, \mathrm{Mn} \\
\mathrm{Cu}, \mathrm{Cd}, \mathrm{Mn}, \mathrm{Zn} \\
\mathrm{Cr}, \mathrm{Cu}, \mathrm{Hg}, \mathrm{Pb}, \mathrm{Ni}, \mathrm{Zn} \\
\mathrm{Cd}, \mathrm{Cr}, \mathrm{Cu}, \mathrm{Hg}, \mathrm{Pb}, \mathrm{Ni}, \mathrm{Zn}\end{array}$} & Alta & $\mathrm{Cd}, \mathrm{Pb}, \mathrm{Hg}, \mathrm{Zn}$ \\
\hline Processos metálicos & & Média & $\mathrm{Co}, \mathrm{Cu}, \mathrm{Ni}$ \\
\hline $\begin{array}{l}\text { pela queima de carvao } \\
\text { Papel } \\
\text { Fertilizantes }\end{array}$ & & Baixa & $\mathrm{Al}, \mathrm{Cr}, \mathrm{Fe}$ \\
\hline
\end{tabular}

Fonte: Adaptado de Volesky (2001).

Muitas tecnologias têm sido desenvolvidas e utilizadas para o tratamento de águas distribuição, entretanto, o alto gasto energético e financeiro empregado, se apresenta como aspecto negativo, já que isso também aumenta o custo de obtenção de água limpa, dificultando sua aquisição pela parcela da população que vive em condições de pobreza, principalmente nos países subdesenvolvidos, onde há escassez de água (REDDY; LEE, 2012).

\section{Mecanismos de (bio)adsorção}

A adsorção é um processo que ocorre quando uma substância (adsorvato), presente em um líquido ou gás, permanece retida sobre a superfície de um sólido (adsorvente), em virtude de interações intermoleculares, eletrostáticas, dipolares, de Van der Waals, ou de um conjunto destas (LAKHERWAL, 2014; BEDIAKO et al., 2015; GHORBEL-ABID; TRABELSI-AYADI, 2015). Essas interações dependem da natureza das espécies envolvidas, podem ocorrer de forma isolada ou simultânea, de modo que quanto mais fortes forem as interações mais efetivo será o processo de adsorção (GHORBEL-ABID; TRABELSI-AYADI, 2015; ANASTOPOULOS; KYZAS, 2015; BEDIAKO et al., 2015). O avanço nas pesquisas com materiais adsortivos permite que a adsorção de metais possa se dá por diferentes vias, a exemplo da troca iônica, interação ácidobase, polarização de eletrônica, forças de dispersão, etc.

Quando se utiliza a biomassa como o adsorvente para retenção, remoção ou recuperação de metais, a técnica passa a ser denominada como biossorção ou sorção, e trata-se de uma tecnologia promissora que busca o desenvolvimento de materiais alternativos, com baixo custo e 
que sejam eficazes quando se objetiva o tratamento de águas residuais (VOLESKY, 2001; ANASTOPOULOS; KYZAS, 2015). Além de exigir pouco processamento, a biomassa é abundante na natureza como resíduo ou subproduto (SAKA; SAHIN; KÜÇÜK, 2012). Atualmente vem sendo testados vários tipos de biomassa para fins de adsorção de metais pesados, incluindo a biomassa fúngica, bacteriana, algas, turfa, madeira, casca, folhas, papel, resíduo de café, entre outros biomateriais (PUJOL et al., 2013; SEN et al., 2015; SIMATE; NDLOVU, 2015; SINGHA; GULERIA, 2015). A efetividade de um biossorvente depende de suas propriedades particulares, como a área superficial, o volume dos poros e sua distribuição de tamanho, bem como dos parâmetros operacionais, como $\mathrm{pH}$, temperatura, concentração das espécies metálicas, tempo de contato e tamanho de partícula do adsorvente (PINO, TOREM, 2011; SAKA; SAHIN; KÜÇÜK, 2012).

\section{Reaproveitamento das cascas da laranja como biossorvente}

A utilização da biomassa vegetal para a adsorção de metais pesados é uma linha de pesquisa promissora, uma vez que diferentes espécies de casca mostraram alta capacidade de remoção de íons metálicos de soluções aquosas (HEGAZI, 2013; SONG et al., 2013; SAMAN et al., 2015), surgindo como uma fonte alternativa à resina de troca iônica e ao carvão ativado (SEN et al., 2015). Além da eficácia para a remoção de íons de metais pesados, mesmo em concentrações mínimas, o baixo custo atrelado à aquisição desses materiais tem atraído à atenção de muitos pesquisadores (FENG, et al., 2011; LASHEEN; AMMAR; IBRAHIM, 2012; NGUYEN et al., 2013; BHATNAGAR; SILLANPÄÄ; WITEK-KROWIAK, 2015). Além disso, a recuperação do metal e regeneração do biossorvente para uma nova aplicação pode ser realizada mediante uma lavagem ácida da casca, tendo em vista que nessa faixa de $\mathrm{pH}$ os grupos carbonilos, carboxilos ou grupos hidroxilos tornam-se protonados, impedindo a atração por íons metálicos (já que estes possuem cargas positivas), liberando os íons metálicos na solução de recuperação (HORSFALL JUNIOR; OGBAN; AKPORHONOR, 2006). Em comparação aos métodos convencionais, esta técnica tem como vantagens: baixo custo operacional, alta eficiência de remoção, minimização química e/ou lodo biológico, regeneração do biossorvente, não exigência de nutrientes adicionais e possibilidade de recuperação de metal (SAKA; SAHIN; KÜÇÜK, 2012; GONÇALVES JÚNIOR, 2013).

Muitos estudos vêm sendo conduzidos com a casca e o mesocarpo de diferentes frutos, como a laranja (LI et al., 2007), o limão (HUSOON; AL-AZZAWI; AL-HIYALY, 2013), banana (WAN NGAH; HANAFIAH, 2008), maracujá (CHAO; CHANG; NIEVA, 2014), fibra de coco verde (SILVA et al., 2013), melancia (LAKSHMIPATHY; SARADA, 2013), maçã (CHAND; BAFANA; PAKADE, 2015), entre outros. A grande ênfase dada à casca da laranja se deve à grande produção e consumo mundial deste fruto, mas que, lamentavelmente, gera um grande acúmulo de lixo orgânico formado, principalmente, pelas cascas. Sem um destino final adequado, esse resíduo constitui uma fonte rica em compostos fenólicos (BHATNAGAR; SILLANPÄ̈̈; WITEK-KROWIAK, 2015), podendo comprometer o ecossistema do corpo receptor.

No Brasil, a laranja (Citrus sinensis) é produzida em grande escala, chegando a 16 milhões de toneladas ao ano (BRASIL, 2014; MAPA, 2015). A fruta pode ser consumida in natura, na forma de sucos ou ainda na preparação de diversos pratos culinários. Os óleos essenciais extraídos de sua casca são utilizados nas indústrias de perfume, cosméticos e de limpeza (ALMEIDA et al., 2012). Atualmente, o país está entre os maiores produtores e exportadores de suco de laranja do mundo (ZULIAN, DÖRR, ALMEIDA, 2013; MAPA, 2015).

A porosidade da biomassa a partir da casca da laranja encontra-se na zona de albedo, sendo essa a parte branca e esponjosa, consistindo em células parenquimáticas alargadas com grandes espaços intercelulares, conforme mostrado na microscopia eletrônica apresentada na Figura 1. No caso da zona de flavedo, a estrutura celular externa é mais compacta, contendo glândulas de óleo e coberta por uma camada de cera natural (LUGO-LUGO, 2009). 


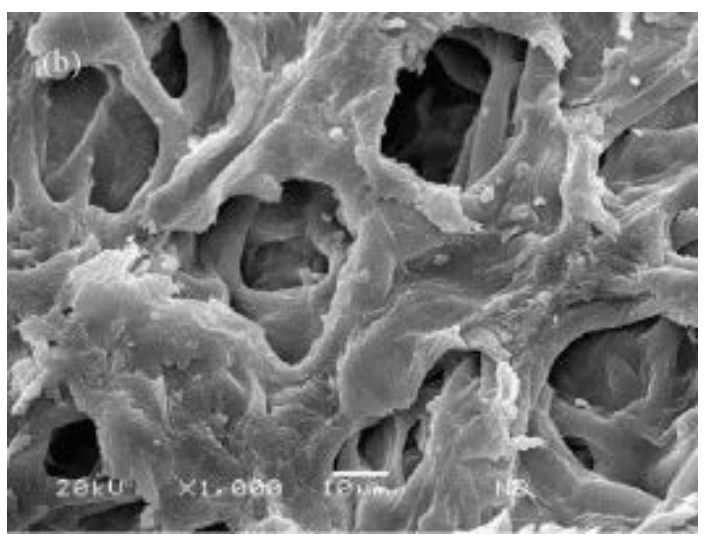

Figura 1- Microfotografia eletrônica de varredura da casca da laranja.

Fonte: Adaptado de Lugo-Lugo et al. (2009).

De um modo geral, a casca da laranja é composta, principalmente, por celulose, pectina, ácido péctico, hemicelulose, lignina, pigmentos de clorofila e outros hidrocarbonetos de baixo peso molecular (XUAN et al., 2006; SAKA; SAHIN; KÜÇÜK, 2012). Esses grupos possuem a capacidade de se ligar aos metais pesados pela doação de um par de elétrons, formando complexos com os íons metálicos em solução (PAGNANELLI et al., 2003). A caracterização desses materiais por espectroscopia na região do infravermelho pode ser visualizada na Figura 2, na qual observa-se uma banda larga e forte em $3429 \mathrm{~cm}^{-1}$ corresponde ao alongamento vibracional da ligação O-H. Já os picos em 1240, 1629 e $1735 \mathrm{~cm}^{-1}$, podem ser atribuídos ao alongamento vibracional da ligação C-O. Identificou-se ainda um pico em $2920 \mathrm{~cm}^{-1}$, característico do alongamento vibracional da ligação C-H. Desta forma, pode-se inferir que há presença da hidroxila, carbonila e dos grupos carboxílicos na casca da laranja. Neste caso, as letras a, b e c, representam a casca da laranja in natura, modificada com $\mathrm{NaOH}$ e modificada com $\mathrm{NaOH}$ e ácido cítrico, respectivamente (SOUZA et al., 2012).

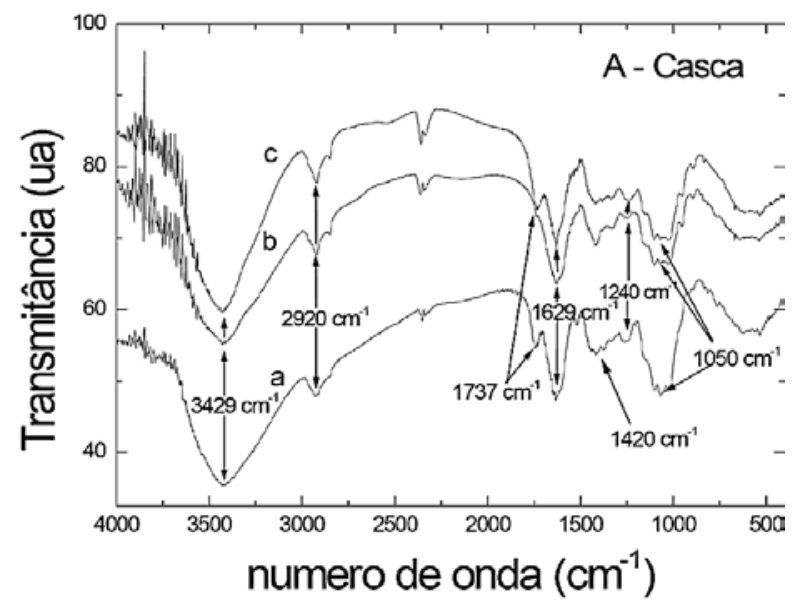

Figura 2- Espectro de infravermelho da casca da laranja. Fonte: Adaptado de Souza et al., (2012).

Outra estratégia de adsorção bastante investigada é a modificação dos biossorventes lignocelulósicos brutos por meio de tratamentos químicos. Este processo visa aumentar a capacidade de sorção. As modificações químicas consistem em fazer a deslignificação de biomassa, a esterificação de grupos carboxílicos e fosfato, metilação dos grupos amino e hidrólise de grupos carboxilato (DEMIRBAS, 2008). Apesar da grande utilização das cascas, ainda há poucas pesquisas que discorram a respeito das características anatômicas e químicas das cascas, bem como sobre o mecanismo do processo de adsorção (RAJA RAO; BHARGAVI, 2013; SEN et al., 2015), impulsionando o investimento de mais pesquisas na área.

Cabe ainda ressaltar que a biossorção não se dá por um único mecanismo, e que os mesmos diferem quantitativamente e qualitativamente, a depender da origem da biomassa, do 
processamento e da espécie utilizada. Podem ocorrer simultaneamente a complexação (formação de um complexo a partir da associação de duas espécies); coordenação (ligação de um átomo central de um complexo com outros átomos por ligação covalente); quelação (união entre complexos formados por um composto orgânico e um metal, por pelo menos dois sítios); troca iônica, (formação de espécies moleculares através do intercâmbio de íons); adsorção (sorção através da superfície do tecido orgânico) e precipitação inorgânica (alteração no meio aquoso levando a uma precipitação do despejo) entre os diferentes cátions e a biomassa (PIETROBELLI, 2007; SILVA et al., 2014).

\section{Estudos de caso}

Estudos foram realizados para a remoção do íon $\mathrm{Cd}^{2+}$ (cádmio) em meio aquoso, modificando quimicamente a casca da laranja após lavagem com álcool isopropílico $\left(\mathrm{C}_{3} \mathrm{H}_{8} \mathrm{O}\right)$, saponificação alcalina $(\mathrm{NaOH})$ e sob diferentes concentração de ácido cítrico $\left(\mathrm{C}_{6} \mathrm{H}_{8} \mathrm{O}_{7}\right)$. Os resultados mostraram que a adsorção foi mais eficiente em $\mathrm{pH}$ 6,0 para os resíduos que foram modificados com o ácido cítrico $\left(0,6 \mathrm{~mol} \mathrm{~L}^{-1}\right)$, sob temperatura de $80^{\circ} \mathrm{C}$ (LI et al., 2007). A adsorção dos íons metálicos na superfície da casca da laranja é possível graças à quantidade de sítios ácidos totais serem maior que os sítios básicos, possibilitando tal interação (VAUGHAN; SEO; MARSHALL, 2001). Nesse caso, o aumento dos sítios ácidos pode ser explicado pela modificação com o ácido cítrico (RANGEL-MENDEZ; STREAT, 2002). O acompanhamento do $\mathrm{pH}$ forneceu as seguintes conclusões: (1) em $\mathrm{pH}$ mais baixos, os íons $\mathrm{H}^{+}$competem com os íons metálicos, ocupando com mais facilidade a superfície dos biossorvente; (2) na faixa de $\mathrm{pH}$ entre 5,8 e 6,5, aumentaram-se as cargas negativas do biossorvente, atraindo os íons metálicos; (3) com o aumento do pH houve a formação de complexos aniônicos de hidróxido, diminuindo a concentração do íons metálicos livres e, consequentemente, sua adsorção. Foi observado ainda que, à medida que o $\mathrm{Cd}^{2+}$ é adsorvido na superfície do biossorvente, há liberação de $\mathrm{H}^{+}$para a solução, o que faz o pH da mesma diminuir.

Um estudo realizado por Lugo-Lugo et al. (2009) para adsorção de $\mathrm{Pb}^{2+}$ com a casca de laranja quimicamente modificada corrobora com os relatos de Sen et al. (2015) em relação ao efeito do $\mathrm{pH}$, na qual, para um valor de $\mathrm{pH}$ 2,0, houve repulsão eletrostática entre a superfície do biossorvente e o íon metálico, em consequência do excesso de íons $\mathrm{H}^{+}$, e à medida que o pH do sistema aumentou, observou-se um favorecimento à adsorção dos metais, que se deu devido a atração eletrostática, sendo que a maior adsorção ocorreu em pH 5,0. Além disso, com o aumento do $\mathrm{pH}$ ocorre um decréscimo de solubilidade dos complexos metálicos e, como além da atração do adsorvato pela superfície do sólido necessita-se que haja um comportamento liofóbico (a sorção aumenta com o decréscimo da solubilidade) para a maioria dos metais, isto significa que a adsorção aumenta com o aumento do pH (SILVA et al., 2014).

Almeida et al. (2012) estudou soluções aquosas de nitrato de chumbo (10 g/L) e nitrato de prata (1 g/L), a fim de observar o percentual de remoção de cada íon a partir da casca da laranja bruta. A remoção de chumbo foi aproximadamente $100 \%$, mas a remoção de prata não foi expressiva. Os resultados atestaram o observado por muitos pesquisadores, onde a capacidade adsortiva de cada material também pode variar de um metal para outro.

Diferentes modificações químicas sob a casca de laranja foram preparadas para entender o mecanismo de adsorção de $\mathrm{Cu}^{2+}$ a partir de soluções de cloreto. A que obteve maior destaque foi a saponificação alcalina (álcool isopropílico aquoso), sob agitação com $\mathrm{NaOH}$ e ácido cítrico $\left(0,6 \mathrm{~mol} \mathrm{~L}^{-1}\right)$ a $80^{\circ} \mathrm{C}$, onde a adsorção máxima de $\mathrm{Cu}^{2+}$ foi de $1,22 \mathrm{~mol} \mathrm{~kg}^{-1}$ (LU et al., 2009).

Em águas residuais, geralmente, existem vários metais disponíveis para a remoção, podendo até ocorrer a supressão da adsorção de algum desses, pois os cátions metálicos irão competir entre si, buscando ocupar os sítios ativos (BULUT; TEZ, 2006; SEN et al., 2015). Estudos realizados com resíduos provenientes da galvanoplastia, contendo $\mathrm{Zn}, \mathrm{Ni}, \mathrm{Cu}, \mathrm{Pb}$ e $\mathrm{Cr}$, mostraram potencial da casca da laranja como adsorvente. A adsorção máxima de Ni (II), Cu (II), $\mathrm{Zn}$ (II) e $\mathrm{Pb}$ (II) chegou a $40 \mathrm{mg} \mathrm{L}^{-1}$, enquanto que a adsorção de Cr (VI) manteve-se mínima ao longo de uma ampla faixa de concentração $\left(20-100 \mathrm{mg} \mathrm{L}^{-1}\right)$, conforme mostra a Figura 3. Em baixas concentrações $\left(20 \mathrm{mg} \mathrm{L}^{-1}\right)$, a ordem de absorção foi $\mathrm{Ni}$ (II)> $\mathrm{Pb}$ (II) $>\mathrm{Zn}$ (II)> Cu (II) > Cr 
(VI). Na concentração mais elevada (100 $\left.\mathrm{mg} \mathrm{L}^{-1}\right)$, a ordem observada foi $\mathrm{Ni}$ (II) $>\mathrm{Cu}$ (II) $>\mathrm{Pb}$ (II) $>$ Zn (II) > Cr (VI) (AJMAL et al., 2000).

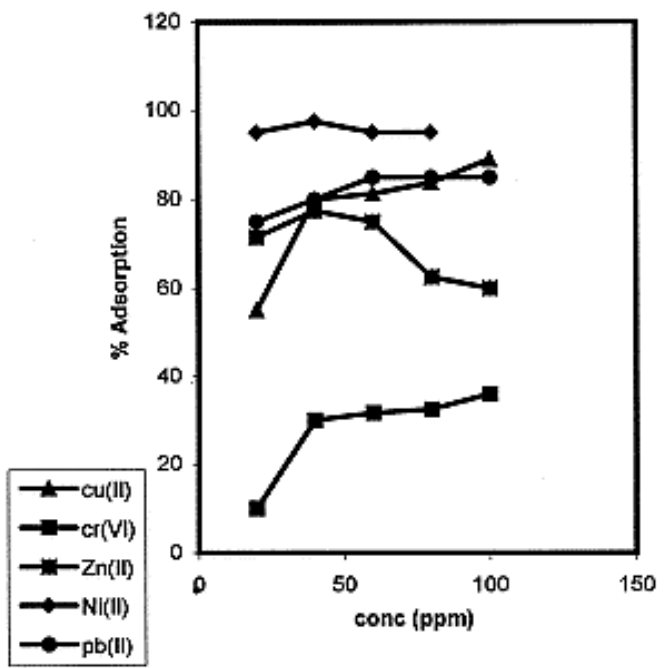

Figura 3 - Efeito da concentração de biossorvente sob a adsorção de metais.

Fonte: Ajmal et al. (2000).

LI et al. (2008) estudaram o efeito de diferentes modificações químicas sobre as propriedades adsorventes, incluindo diferentes saponificações alcalinas $\left(\mathrm{NaOH}, \mathrm{NH}_{4} \mathrm{OH}\right.$ e $\left.\mathrm{Ca}(\mathrm{OH})_{2}\right)$, ácidos diferentes $\left(\mathrm{C}_{6} \mathrm{H}_{6} \mathrm{O}_{7} \cdot \mathrm{H}_{2} \mathrm{O}, \mathrm{H}_{2} \mathrm{C}_{2} \mathrm{O}_{4}\right.$ e $\left.\mathrm{H}_{3} \mathrm{PO}_{4}\right)$ e modificação após saponificação com $\mathrm{NaOH}$ (SNA), foram investigados para adsorção dos íons Ni (II), Co (II), Zn (II) e Cd (II). Por meio dos espectros de FTIR, percebeu-se que existem diferentes grupos funcionais em adsorventes que são capazes de reagir com os íons de metal em solução aquosa. As capacidades de adsorção máxima de Ni (II), Co (II), Zn (II) e Cd (II) para o SPA (casca de laranja modificada com ácido fosfórico após SNA), SCA (casca de laranja modificada com ácido cítrico após SNA) e SOA (casca de laranja modificada com ácido oxálico após SNA) aumentaram 95\%, 178\%, 60\% e $130 \%$, respectivamente, em comparação com casca de laranja bruta. O mecanismo de troca iônica proposto para a adsorção de $\mathrm{Na}^{+}, \mathrm{NH}_{4}{ }^{+}, \mathrm{Ca}^{2+}$ e outros cátions bivalentes, pode ser visualizado na Figura 4.

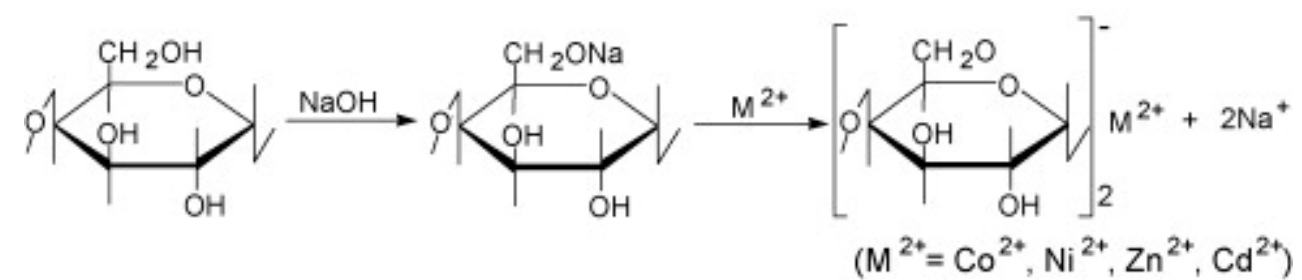

Figura 4 - Proposição mecanística para o processo de adsorção de íons metálicos, utilizando casacas de laranja com diferentes modificações como biossorvente.

Fonte: Li et al. (2008).

Após a saponificação alcalina, os íons de hidrogênio são liberados pra solução e, o $\mathrm{Na}^{+}$ por sua vez, se liga à superfície do adsorvente. Ao se submeter o biossorvente a uma solução com certa concentração de diferentes metais, o $\mathrm{Na}^{+}$é substituído devido à maior afinidade eletrônica destes pela superfície do biossorvente.

Apesar de o carvão ativado comercial ser considerado um ótimo adsorvente de poluentes em águas e efluentes, o alto custo financeiro e energético, bem como a perda da sua capacidade, após ciclos de adsorção-dessorção, limita o seu uso, tornando os biomateriais mais atrativos (BILAL et al. 2013; BHATNAGAR; SILLANPÄÄ; WITEK-KROWIAK, 2015).

Finalmente, fazendo-se uma avaliação geral, percebe-se que as cascas de laranja podem ser utilizadas para substituir o carvão ativado comercial, bem como outros materiais utilizados para tal finalidade, possuindo eficácia igual ou superior aos mesmos, mesmo em concentrações 
traço do metal. Essa capacidade adsortiva pode apresentar melhorias significativas pela modificação da biomassa vegetal, mas a toxicologia desses materiais para o meio ambiente, após processo de saturação, ainda precisa ser investigada.

\section{Considerações finais}

A aplicação da casca da laranja como adsorvente agrega valor a mesma e, contribui para o crescimento da economia local. Este biossorvente apresenta elevada eficiência para a remoção de diferentes metais, com atenção especial para metais pesados como $\mathrm{Zn}, \mathrm{Ni}, \mathrm{Cu}, \mathrm{Cr}, \mathrm{Cd}$, Co e $\mathrm{Pb}$, em diferentes concentrações, demonstrando remoção igual ou semelhante ao carvão ativado comercial. A modificação do biomaterial pode promover grandes melhorias no processo de troca iônica. Os resultados promissores faz da biossorção uma área em constante ascensão.

\section{Orange peel biosorbent potential as an alternative for removing heavy metals from wastewater}

Albstract: The pollution of water resources and the environmental impacts are a global concern, due to the rising scarcity of this resource associated to the high energetic and financial costs associated to their treatment. One of the most worrying and frequent pollutants are heavy metals, because they are highly toxic for living beings and capable of make the water unusable for different purposes. Alternatively, several treatment techniques have been proposed for removal of natural waters metals, highlighting the adsorption technologies for ion exchange. The use of the biosorbents derived from mesocarp of the shell of different fruits (orange, lemon, banana, coconut fiber, among others), have shown very promising results. This paper presents a review of using orange peel viability in the biosorption process, due to the high potential of its fruit inedible biomass for the ion exchange process. In general, studies show that orange peel can be used to replace the activated charcoal trade, that has a high cost, but it is still the most used technique for the removal of metals from contaminated waters. The chemical modification of the orange peel has also shown excellent results for the adsorption of metal ions, however toxicological studies still need to be made about the contamination risks by secondary compounds.

Keywords: Heavy metals; Ion exchange; Orange peel; Biosorption.

\section{Referências bibliográficas}

AJMAL, M. et al. Adsorption studies on Citrus reticulata (fruit peel of orange): removal and recovery of Ni II from electroplating wastewater. Journal of Hazardous Materials, v. 79, n. 12, p 117-131, 2000.

AKAR, S. T. et al. Removal of copper(II) ions from synthetic solution and real wastewater by the combined action of dried Trametes versicolor cells and montmorillonite. Hydrometallurgy, v. 97, n. 1-2, p. 98-104, 2009.

ALMEIDA, J. da S. M de, et al. Redução do teor de prata e chumbo de águas contaminadas através do uso de material adsorvente. Revista Ciências do Ambiente On-Line, v. 8, n. 1, p. 16, 2012.

ALOMÁ-VICENTE, I. de la. C. et al. Panorama general en torno a la contaminación del agua por níquel. La biosorción como tecnología de tratamento. Revista Cubana de Química, v. 25, n. 3, p. 266-280, 2013.

ANASTOPOULOS, I.; KYZAS, G. Z. Progress in batch biosorption of heavy metals onto algae. Journal of Molecular Liquids, v. 209, n. 1, p. 77-86, 2015. 
BEDIAKO, J. K. et al. Removal of heavy metals from aqueous phases using chemically modified waste Lyocell fiber. Journal of Hazardous Materials, v. 299, n. 1, p. 550-561, 2015.

BHATNAGAR, A.; SILLANPÄÄ, M.; WITEK-KROWIAK, A. Agricultural waste peels as versatile biomass for water purification - A review. Chemical Engineering Journal, v. 270, n. 1, p. 244-271, 2015.

BILAL, M. et al. Waste biomass adsorbents for copper removal from industrial wastewater - A review. Journal of Hazardous Materials, v. 263, n. 1, p. 322-333, 2013.

BRASIL. Ministério da Agricultura, Pecuária e Abastecimento. Projeções do Agronegócio: Brasil 2013/2014 a 2023/2024 projeções de longo prazo, Brasília, DF, set., 2014. 100p.

Disponível em: <http://www.agricultura.gov.br/arq_editor/file/projecoes_2013-2014_20232024.pdf $>$. Acesso em: 15 set. 2015.

BULUT, Y.; TEZ, Z. Removal of heavy metals from aqueous solution by sawdust adsorption. Journal of Environmental Sciences, v. 19, n. 2, p. 160-166, 2007.

CAPRARESCU, S. et al. Adsorbents/ion exchangers-PVA blend membranes: Preparation, characterization and performance for the removal of $\mathrm{Zn}^{2+}$ by electrodialysis. Applied Surface Science, v. 329, n. 1, p. 65-75, 2015.

CHAND, P.; BAFANA, A.; PAKADE, Y. B. Xanthate modified apple pomace as an adsorbent for removal of $\mathrm{Cd}$ (II), $\mathrm{Ni}$ (II) and $\mathrm{Pb}$ (II), and its application to real industrial wastewater.

International Biodeterioration \& Biodegradation, v. 97, n. 1, 60-66, 2015.

CHAO, H. P.; CHANG, C. C.; NIEVA, A. Biosorption of heavy metals on Citrus maxima peel, passion fruit shell, and sugarcane bagasse in a fixed-bed column. Journal of Industrial and Engineering Chemistry, v. 20, n. 5, p. 3408-3414, 2014.

DEMIRBAS, A. Heavy metal adsorption onto agro-based waste materials: A review. Journal of Hazardous Materials, v. 157, n. 2-3, p. 220-229, 2008.

FENG, N. et al. Biosorption of heavy metals from aqueous solutions by chemically modified orange peel. Journal of Hazardous Materials, v. 185, n. 1, p. 49-54, 2011.

FU, F.; WANG, Q. Removal of heavy metal ions from wastewaters: A review. Journal of Environmental Management, v. 92, n. 3, 407-418, 2011.

GONÇALVES JÚNIOR, A. C. Descontaminação e monitoramento de águas e solos na região amazônica utilizando materiais adsorventes alternativos, visando a remoção de metais pesados tóxicos e pesticidas. Inclusão Social, Brasília, v. 6, n. 2, p. 105-113, 2013.

GHORBEL-ABID, I.; TRABELSI-AYADI, M. Competitive adsorption of heavy metals on local landfill clay. Arabian Journal of Chemistry, v. 8, n. 1, p. 25-31, 2015.

HE, J.; CHARLET, L. A review of arsenic presence in China drinking water. Journal of Hydrology, v. 492, n. 1, p. 79-88, 2013.

HEGAZI, H. A. Removal of heavy metals from wastewater using agricultural and industrial wastes as adsorbents. HBRC Journal, v. 9, n. 3, p. 276-282, 2013.

HORSFALL JUNIOR, M.; OGBAN, F. E.; AKPORHONOR, E. E. Recovery of lead and cadmium ions from metal-loaded biomass of wild cocoyam (Caladium bicolor) using acidic, basic and neutral eluent solutions. Electronic Journal of Biotechnology, v. 9, n. 2, p. 152-156, 2006.

HUSOON, Z. A.; AL-AZZAWI, M. N. A.; AL-HIYALY, S. A. K. Investigation biosorption potential of copper and lead from industrial wastewater using orange and lemon peels. Journal of Al-Nahrain University Science, v. 16, n. 2, p. 173-179, 2013.

KEDZIOREK, M. A. M. et al. Natural attenuation of heavy metals (Cd, $\mathrm{Cr}$, and $\mathrm{Pb}$ ) in a water table aquifer underlying an industrial site. Procedia Earth and Planetary Science, v. 7, n. 1, p. 89-92, 2013. 
KUSHWAHA, S. et al. Spectroscopic characterization for remediation of copper, cadmium and mercury using modified palm shell powder. Journal of the Taiwan Institute of Chemical Engineers, v. 46, n. 1, p. 191-199, 2015.

KYZAS, G. Z.; KOSTOGLOU, M. Green adsorbents for wastewaters: A critical review. Materials, v. 7, n. 1, p. 333-364, 2014.

LAKHERWAL, D. Adsorption of heavy metals: A review. International Journal of Environmental Research and Development. v. 4, n. 1, p. 41-48, 2014.

LAKSHMIPATHY, R.; SARADA, N. C. Application of watermelon rind as sorbent for removal of nickel and cobalt from aqueous solution. International Journal of Mineral Processing, v. 122, n. 1, p. 63-65, 2013.

LASHEEN, M. R.; AMMAR, N. S.; IBRAHIM, H. S. Adsorption/desorption of Cd(II), Cu(II) and Pb(II). Solid State Sciences, v. 14, n. 2, p. 202-210, 2012.

LI, X. et al. Study on the preparation of orange peel cellulose adsorbents and biosorption of $\mathrm{Cd}^{2+}$ from aqueous solution. Separation and Purification Technology, v. 55, n. 1, p.69-75, 2007.

LI, X. et al. Preparation and evaluation of orange peel cellulose adsorbents for effective removal of cadmium, zinc, cobalt and nickel. Colloids and Surfaces A: Physicochemical and Engineering Aspects, v. 317, n. 1-3, p. 512-521, 2008.

$\mathrm{LU}$, D. et al. Kinetics and equilibrium of $\mathrm{Cu}(\mathrm{II})$ adsorption onto chemically modified orange peel cellulose biosorbents. Hydrometallurgy, v. 95, n. 1-2, p. 145-152, 2009.

LUGO-LUGO, V. et al. A comparative study of natural, formaldehyde-treated and copolymer grafted orange peel for $\mathrm{Pb}(\mathrm{II})$ adsorption under batch and continuous mode. Journal of Hazardous Materials, v. 161, n. 2-3, p. 1255-1264, 2009.

MAPA- Ministério da Agricultura, Pecuária e Abastecimento. Citrus. Disponível em: <http://www.agricultura.gov.br/vegetal/culturas/citrus>. Acesso em: 15 set. 2015.

MOREIRA, F. R.; MOREIRA, J. C. Os efeitos do chumbo sobre o organismo humano e seu significado para a saúde. Rev Panam Salud Publica, v. 15, n. 2, p. 119-129, 2004.

NGUYEN, T. A. H. et al. Applicability of agricultural waste and by-products for adsorptive removal of heavy metals from wastewater. Bioresource Technology, v. 148, n. 1, p. 574-585, 2013.

PAGNANELLI, F. et al. Heavy metal removal by olive pomace: biosorbent characterisation and equilibrium modelling. Chemical Engineering Science, v. 58, n. 20, p. 4709- 4717, 2003.

PIETROBELLI, J. M. T. de A. Avaliação do potencial de biossorção dos íons Cd (II), Cu (II) e Zn (II) pela macrófita Egeria Densa. 2007. 98f. Dissertação (Mestrado em Engenharia Química) - Universidade Estadual do Oeste do Paraná, Toledo, 2007.

PINO, G. H. TOREM, M. L. Aspectos fundamentais da biossorção de metais não ferrosos Estudo de caso. Tecnologia em Metalurgia, Materiais e Mineração., v. 8, n. 1, p. 57-63, 2011.

PUJOL, D. et al. Modelling synergistic sorption of $\mathrm{Cr}(\mathrm{VI}), \mathrm{Cu}(\mathrm{II})$ and $\mathrm{Ni}(\mathrm{II})$ onto exhausted coffee wastes from binary mixtures $\mathrm{Cr}(\mathrm{VI})-\mathrm{Cu}(\mathrm{II})$ and $\mathrm{Cr}(\mathrm{VI})-\mathrm{Ni}(\mathrm{II})$. Chemical Engineering Journal, v. 230, n. 1, p. 396-405, 2013.

RAJA RAO, P.; BHARGAVI, C. H. Studies on biosorption of heavy metals using pretreated biomass of fungal species. International Journal of Chemistry and Chemical Engineering, v. 3, n. 3, p. 171-180, 2013.

RANGEL-MENDEZ, J. R.; STREAT, M. Adsorption of cadmium by activated carbon cloth: influence of surface oxidation and solution pH. Water Research, v. 36, n. 5, p. 1244-1252, 2002. 
RAZAK, N. H. A. et al. Drinking water studies: A review on heavy metal, application of biomarker and health risk assessment (a special focus in Malaysia). Journal of Epidemiology and Global Health, 2015, http://dx.doi.org/10.1016/j.jegh.2015.04.003.

REDDY, D. H. K.; LEE, S. M. Water pollution and treatment technologies. Environmental \& Analytical Toxicology, v. 2, n. 5, p. 1-2, 2012.

SAKA, C.; SAHIN, O.; KÜÇÜK, M. M. Applications on agricultural and forest waste adsorbents for the removal of lead (II) from contaminated waters. International Journal of Environmental Science and Technology, v. 9, n. 2, p. 379-394, 2012.

SAMAN, N. et al. Adsorptive efficacy analysis of lignocellulosic waste carbonaceous adsorbents toward different mercury species. Process Safety and Environmental Protection, v. 96, n. 1, p. 33-42, 2015.

SEN, A. et al. Heavy metals removal in aqueous environments using bark as a biosorbent. International Journal of Environmental Science and Technology, v. 12, n. 1, p. 391-404, 2015.

SILVA, J. L. B. C. da, et al. Biossorção de metais pesados: Uma revisão. Revista Saúde e Ciência On line, v. 3, n. 3, p. 137-149, 2014.

SILVA, K. M. D. da, et al. Caracterização físico-química da fibra de coco verde para a adsorção de metais pesados em efluente de indústria de tintas. Engevista, v. 15, n. 1. p. 43-50, 2013.

SIMATE, G. S.; NDLOVU, S. The removal of heavy metals in a packed bed column using immobilized cassava peel waste biomass. Journal of Industrial and Engineering Chemistry, v. 21, n. 1, p. 635-643, 2015. .

SINGHA, A. S.; GULERIA, A. Utility of chemically modified agricultural waste okra biomass for removal of toxic heavy metal ions from aqueous solution. Engineering in Agriculture, Environment and Food, v. 8, n. 1, p. 52-60, 2015.

SONG, S. T. et al. Removal of Hg(II) from aqueous solution by adsorption using raw and chemically modified rice straw as novel adsorbents. Industrial \& Engineering Chemistry Research, v. 52, n. 36, p. 13092-13101, 2013.

SOUZA, J. V. T. M. de, et al. Adsorção de cromo (III) por resíduos de laranja in natura e quimicamente modificados. Semina: Ciências Exatas e Tecnológicas, v. 33, n. 1, p. 03-16, 2012.

VAUGHAN, T.; SEO, C. W.; MARSHALL, W. E. Removal of selected metal ions from aqueous solution using modified corncobs. Bioresource Technology, v. 78, n. 2, p. 133-139, 2001.

VOLESKY, B. Detoxification of metal-bearing effluents: biosorption for the next century. Hydrometallurgy, v. 59, n. 2-3, 203-216, 2001.

WAN NGAH, W. S.; HANAFIAH, M. A. K. M. Removal of heavy metal ions from wastewater by chemically modified plant wastes as adsorbents: A review. Bioresource Technology, v. 99, n. 10, p. 3935-3948, 2008.

WU, M. D. J. et al. Effect of low-level prenatal mercury exposure on neonate neurobehavioral development in China. Pediatric Neurology, v. 51, n. 1, p. 93-99, 2014.

XUAN, Z. et al. Study on the equilibrium, kinetics and isotherm of biosorption of lead ions onto pretreated chemically modified orange peel. Biochemical Engineering Journal, v. 31, n. 2, p. 160-164, 2006.

ZULIAN, A.; DÖRR, A. C.; ALMEIDA, S. C. Citricultura e agronegócio cooperativo no Brasil. Revista Eletrônica em Gestão, Educação e Tecnologia Ambiental, v. 11, n. 11, p. 2290 2306, 2013. 